\title{
Gelatin and Starch: What Better Stabilizes the Enzyme Activity?
}

\author{
E. N. Esimbekova ${ }^{a, b, *}$, A. E. Govorun ${ }^{b}$, V. I. Lonshakova-Mukina ${ }^{b}$, and V. A. Kratasyuk ${ }^{a, b}$ \\ Presented by Academician A.G. Degermendzhi
}

Received November 30, 2019; revised November 30, 2019; accepted November 30, 2019

\begin{abstract}
The regularities of the functioning of a number of enzymes in a viscous environment created by natural polymers, starch and gelatin are examined. Based on the analysis of kinetic curves of thermal inactivation, mechanisms of thermal inactivation of enzymes in a viscous microenvironment are proposed. Using the example of butyrylcholinesterase, NAD(P)H:FMN oxidoreductase, and coupled system of the luminous bacteria (NAD(P)H:FMN oxidoreductase + luciferase), the conditions, under which starch and gelatin have a stabilizing effect on enzyme activity during storage and exposure to various physical and chemical environmental factors, were found. A significant increase in the stabilizing effect is achieved by eliminating water during drying the enzyme preparations immobilized in starch and gelatin polymer gels.
\end{abstract}

Keywords: enzyme stabilization, thermal inactivation of enzymes, starch, gelatin, butyrylcholinesterase, luciferase, NAD(P)H:FMN oxidoreductase

DOI: $10.1134 / \mathrm{S} 0012496620020039$

The low stability of protein macromolecules of enzymes limits the scope of their practical application. In this regard, the search for possible ways to increase the stability of enzymes is an important task. In this case, two different aspects of the problem are taken into account: thermodynamic (or conformational) stability and kinetic (or long-term) stability. The addition of stabilizers is one of the ways to increase the stability of enzymes [1]. Immobilization is a versatile method used to increase both thermodynamic and kinetic stability. At the same time, in order to stabilize different enzymes, it is necessary to select their unique microenvironment. In recent years, studies of the stabilization of protein macromolecules under the conditions of molecular crowding, i.e., under the conditions of the excluded volume of surrounding free water, have become popular [2-4]. Stabilization of protein molecules when immobilized in polymer gels and placed in media with increased viscosity is achieved precisely due to the aforementioned effect [5]. To date, the physicochemical and structural properties of gels and viscous solutions of natural biopolymers, gelatin and starch, have been studied quite well [6, 7]. In addition,

\footnotetext{
${ }^{a}$ Institute of Biophysics, Siberian Branch, Russian Academy of Sciences, Krasnoyarsk, 660036 Russia

${ }^{b}$ Siberian Federal University, Krasnoyarsk, 660041 Russia

*e-mail: esimbekova@yandex.ru
}

there is evidence of a favorable effect of the microenvironment created by these biopolymers on enzyme molecules [8-10].

In this study, we used the enzymes butyrylcholinesterase (BChE), NAD(P)H:FMN oxidoreductase (Red), and the NAD(P)H:FMN oxidoreductase and luciferase (Red + Luc) coupled enzyme system of the luminous bacteria as examples to analyze the peculiarities of the functioning of enzymes when placed in viscous solutions and gels of natural biopolymers, starch and gelatin. It has been shown that under certain conditions starch and gelatin are able to exert a stabilizing effect on the functioning of enzymes during storage and exposure to various physical and chemical factors of the environment. The results obtained are important for the development of highly stable enzyme preparations for application in biological analyzes, in particular, biotesting of natural water, wastewaters, and aqueous solutions.

Here we used the following reagents: FMN (Serva, Germany), NADH (Gerbu, Germany), tetradecanal (Merck, Germany), S-butyrylthiocholine iodide and 5-5'-dithiobis(2-nitrobenzoic acid) (Sigma, Germany), lyophilized preparations NAD $(\mathrm{P}) \mathrm{H}: \mathrm{FMN}$ oxidoreductase (EC 1.5.1.29) from Vibrio fischeri, enzyme system consisting of $\mathrm{NAD}(\mathrm{P}) \mathrm{H}: \mathrm{FMN}$ oxidoreductase and luciferase (EC 1.14.14.3) from a recombinant $E$. coli strain (manufactured by the Lab- 

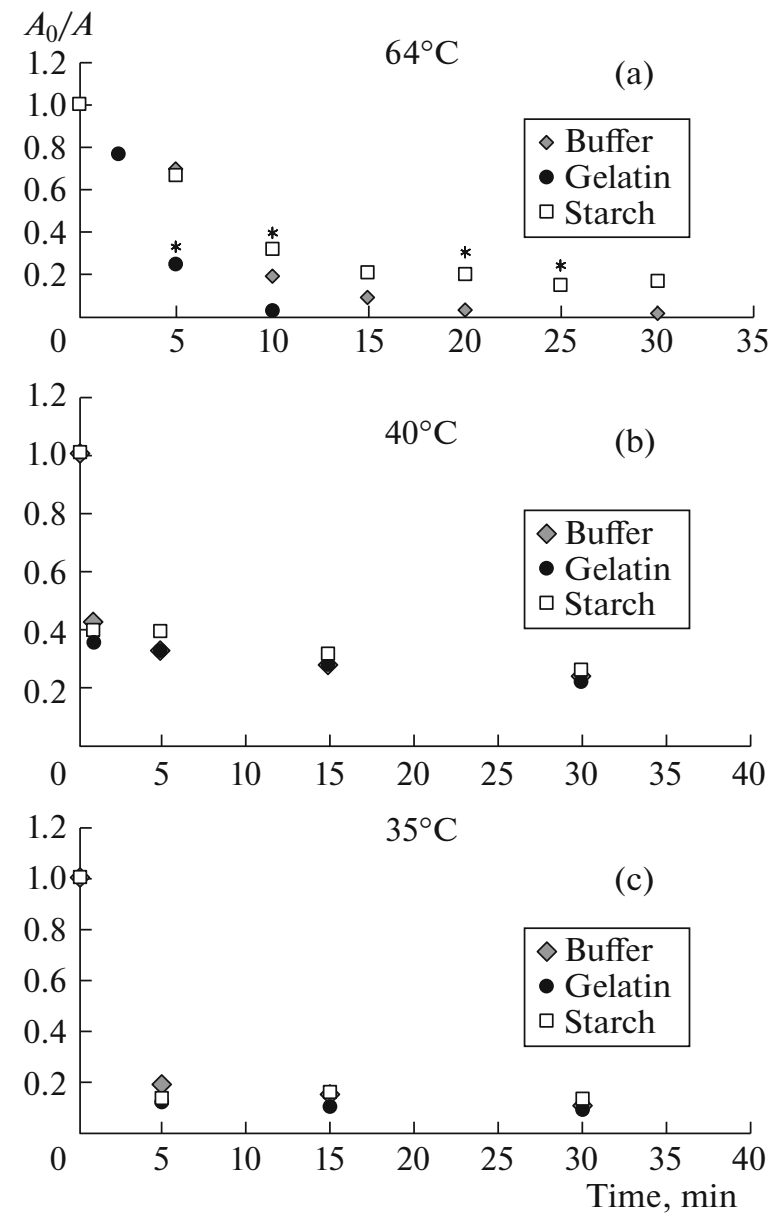

Fig. 1. Dependences of the residual activity of enzymes on the time of incubation in a buffer solution without additives and with the addition of starch and gelatin: (a) BChE (gelatin and starch concentrations are 1.4 and 3\%, respectively); (b) Red (gelatin and starch concentrations are 1 and $2 \%$, respectively); (c) Red + Luc (gelatin and starch concentrations are 0.5 and $2 \%$, respectively); ${ }^{*} p<0.05$ compared to residual activity in the buffer.

oratory of Nanobiotechnology and Bioluminescence of the Institute of Biophysics of the Siberian Branch of the Russian Academy of Sciences, Krasnoyarsk), and butyrylcholinesterase from horse serum (Sigma, Germany).

The activity of Red was determined spectrophotometrically by the rate of NADH consumption [11]. The activity of the Red + Luc coupled enzyme system was determined by the value of the maximum luminescence intensity $I_{\max }$ expressed in relative units [10]. The activity of BChE was determined as the rate of hydrolysis of the substrate S-butyrylthiocholine iodide in a solution containing 5-5'-dithiobis(2-nitrobenzoic acid) [12].

The kinetic dependences of the residual enzyme activity $\left(A / A_{0}\right)$ were obtained during incubation of enzymes in $0.05 \mathrm{M}$ potassium phosphate buffer solu-

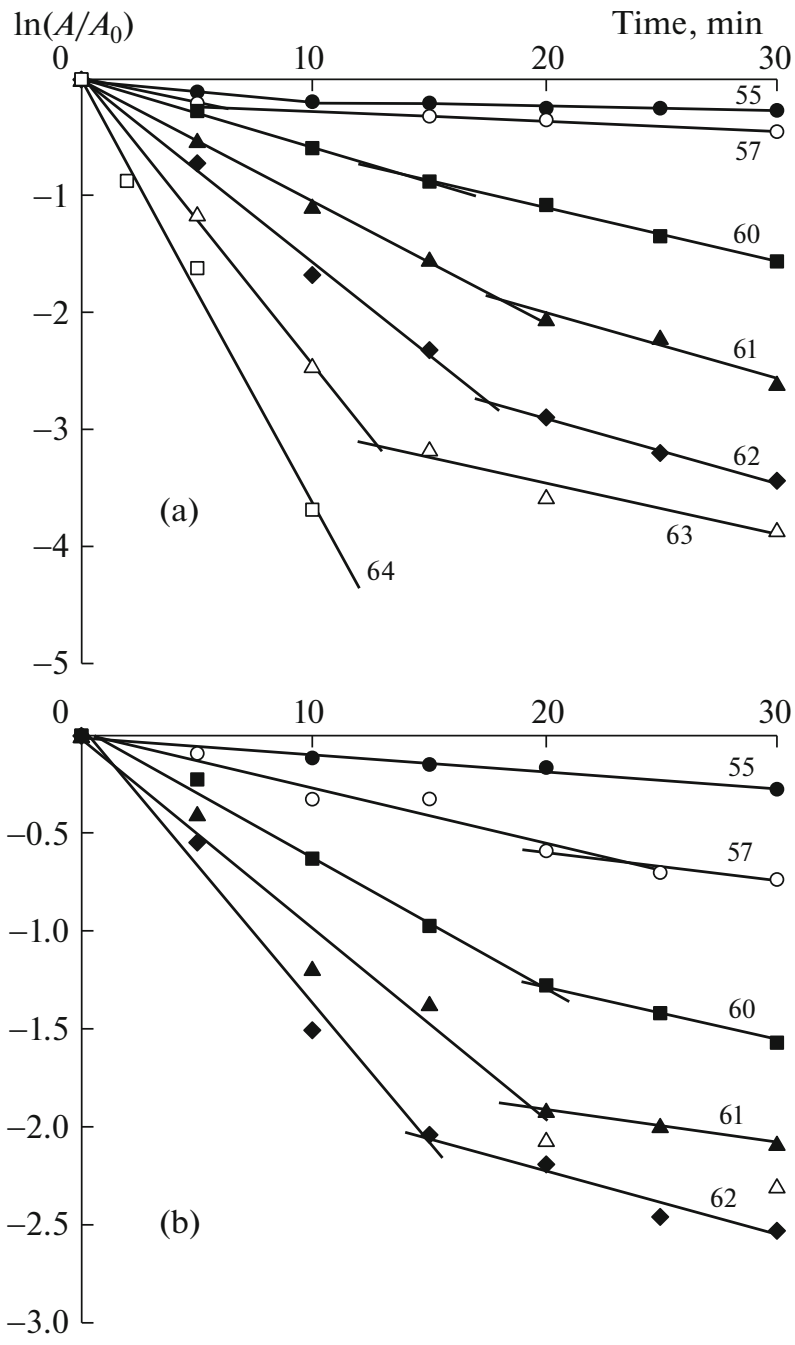

Fig. 2. Kinetic dependences of the thermal inactivation of $\mathrm{BChE}$ in (a) a $1.4 \%$ gelatin solution and (b) a $3 \%$ starch solution at various temperatures.

tion without additives for 0 to $30 \mathrm{~min}$, as well as in starch and gelatin buffer solutions at various temperatures (Fig. 1). It is seen that the starch solution has a greater stabilizing effect on $\mathrm{BChE}$ compared to the gelatin solution. For example, the residual activity of $\mathrm{BChE}$ is retained in a starch environment at a temperature of $64^{\circ} \mathrm{C}$ for $30 \mathrm{~min}$, and in a gelatin environment for $10 \mathrm{~min}$ (Fig. 1a). At the same time, the residual activity of Red and Red + Luc during incubation of enzymes in viscous starch and gelatin solutions at various temperatures does not differ from that in the buffer solution (Figs. 1b, 1c).

Based on the obtained experimental data, kinetic curves of the thermal inactivation of enzymes in a buffer solution and viscous solutions of starch and gelatin were constructed. All the obtained dependences in the coordinates of the first-order equation have the form of curves with a "kink," which is characteristic of the 
Table 1. Effective rate constants of the first $\left(k_{1}, \mathrm{~min}^{-1}\right)$ and second $\left(k_{2}, \mathrm{~min}^{-1}\right)$ stages of temperature inactivation of BChE in a buffer solution without additives (control) and with the addition of gelatin and starch at different temperatures $(M \pm m$, $n=3)$

\begin{tabular}{c|c|c|c|c|c|c}
\hline \multirow{2}{*}{$T,{ }^{\circ} \mathrm{C}$} & \multicolumn{2}{|c|}{ Buffer } & \multicolumn{2}{c|}{$1.4 \%$ gelatin } & \multicolumn{2}{c}{$3 \%$ starch } \\
\cline { 2 - 6 } & $k_{1} \times 10^{3}$ & $k_{2} \times 10^{3}$ & $k_{1} \times 10^{3}$ & $k_{2} \times 10^{3}$ & $k_{1} \times 10^{3}$ & $k_{2} \times 10^{3}$ \\
\hline 55 & $47 \pm 2$ & $19 \pm 1$ & $19.5 \pm 1.6^{*}$ & $3.8 \pm 0.6^{*}$ & $12 \pm 2^{*}$ & $11.0 \pm 1.4^{*}$ \\
57 & $58 \pm 2$ & $32 \pm 3$ & $38 \pm 2^{*}$ & $9.0 \pm 0.8^{*}$ & $24 \pm 3^{*}$ & $7.7 \pm 1.3^{*}$ \\
60 & $53 \pm 3$ & $53 \pm 4$ & $49 \pm 5$ & $46 \pm 7$ & $67 \pm 9^{*}$ & $28 \pm 3^{*}$ \\
61 & $114 \pm 5$ & $48 \pm 5$ & $104 \pm 5$ & $56 \pm 5$ & $98 \pm 8^{*}$ & $17 \pm 2^{*}$ \\
62 & $185 \pm 18$ & $47 \pm 20$ & $158 \pm 11$ & $110 \pm 8^{*}$ & $142 \pm 18^{*}$ & $33 \pm 3$ \\
64 & $174 \pm 25$ & $92 \pm 7$ & $456 \pm 50^{*}$ & - & $111 \pm 9^{*}$ & $21 \pm 2^{*}$ \\
\hline
\end{tabular}

$* p<0.05$ in comparison with the control values at the corresponding temperature; - , the pseudo-first order of the temperature inactivation rate constant is observed in gelatin for a given temperature.

dissociative mechanism of thermal inactivation of oligomeric enzymes [13]. The initial segments of the kinetic curves correspond to the process of the enzyme dissociation into subunits ("fast inactivation"); the second linear sections, into the denaturation of individual subunits ("slow inactivation"). As an example, Fig. 2 shows the kinetic curves for the thermal inactivation of $\mathrm{BChE}$ in gelatin and starch solutions at various temperatures. Similar dependences were obtained for Red and the Red + Luc coupled enzyme system.

The effective thermal inactivation rate constants $k_{1}$ and $k_{2}$ for the "fast" and "slow" stages of enzyme inactivation, respectively, which are the slope of the thermal inactivation curves, were calculated from the kinetic dependences of the thermal inactivation of the enzymes. The $k_{1}$ and $k_{2}$ values obtained by analyzing the BChE thermal inactivation curves are shown in Table 1 for example. It is seen that the stabilizing effect of starch is expressed by the slowdown of both the first and second stages of inactivation of the enzyme. For Red and Red + Luc, no significant differences were found between the $k_{1}$ and $k_{2}$ values for the enzymes that function in the buffer and solutions of starch or gelatin.

Thus, among the studied enzyme systems, an increase in thermodynamic stability during exposure to high temperatures $\left(>60^{\circ} \mathrm{C}\right)$ was observed only for $\mathrm{BChE}$ in a starch solution.

Comparison of the obtained results with published data indicates that the thermodynamic and kinetic stability of enzymes can be enhanced by the introduction of an additional procedure for drying the enzymes during their immobilization in starch and gelatin gels. Thus, it has been shown that drying of the Red + Luc coupled enzyme system immobilized in starch and gelatin gels significantly increases its resistance to chemical and physical environmental factors: an expansion of the $\mathrm{pH}$ optimum to the acidic and alkaline regions is observed; high enzyme activity is retained with increasing salt concentrations; thermal stability is increased [14]. It has been shown that enzyme preparations being dried starch or gelatin disks possess high stability during storage: the activity of BChE preparations as dried starch disks lasted more than 300 days [12]; the activity of Red + Luc preparations in the form of gelatin disks, at least two years [10].

Thus, to achieve the thermodynamic and kinetic stability of the enzymes, it is more preferable to use starch as a stabilizing additive. Based on the totality of the presented results and literature data, it can be concluded that the key factor in the stabilization of enzymes by incorporation in the starch and gelatin polymer gels is the exclusion of water when drying immobilized enzyme preparations. The results are the basis for obtaining highly active and stable enzyme preparations for biological analyzes.

\section{FUNDING}

The reported study was funded by Russian Foundation for Basic Research, Government of Krasnoyarsk Territory, Krasnoyarsk Regional Fund of Science, project nos. 18-44243010 and 18-44-242003.

\section{COMPLIANCE WITH ETHICAL STANDARDS}

The authors declare that they have no conflict of interest. This article does not contain any studies involving animals or human participants performed by any of the authors. 


\section{REFERENCES}

1. Lonshakova-Mukina, V., Esimbekova, E., and Kratasyuk, V., Sen. Actuat., 2015, vol. 213, pp. 244-247.

2. Cheung, M.S., Klimov, D., and Thirumalai, D., Proc. Natl. Acad. Sci. U.S.A., 2005, vol. 102, pp. 4753-4758.

3. Stagg, L., Zhang, S.Q., Cheung, M.S., et al., Proc. Natl. Acad. Sci. U.S.A., 2007, vol. 104, pp. 1897618981.

4. Makowski, L., Rodi, D.J., Mandava, S., et al., J. Mol. Biol., 2008, vol. 375, no. 2, pp. 529-546.

5. Ragoonanan, V. and Aksan, A., Transf. Med. Hemother., 2007, vol. 34, pp. 246-252.

6. Djabourov, M., Cont. Phys., 1988, vol. 29, no. 3, pp. 273-297.

7. Copeland, L., Blazek, J., Salman, H., et al., Food Hydrocoll., 2009, vol. 23, pp. 1527-1534.

8. Munjal, N. and Sawhney, S.K., Enz. Microb. Technol., 2002, vol. 30, no. 5, pp. 613-619.
9. Hoffmann, I., Silva, V.D., and Nascimento, M.G., J. Braz. Chem. Soc., 2011, vol. 22, no. 8, pp. 1559-1567.

10. Bezrukikh, A., Esimbekova, E., Nemtseva, E., et al., Anal. Bioanal. Chem., 2014, vol. 406, no. 23, pp. 57435747.

11. Govorun, A.E., Esimbekova, E.N., and Kratasyuk, V.A., Dokl. Biochem. Biophys., 2019, vol. 486, pp. 213-215.

12. Lonshakova-Mukina, V.I., Esimbekova, E.N., and Kratasyuk, V.A., Dokl. Biochem. Biophys., 2018, vol. 479, pp. $98-100$.

13. Poltorak, O.M. and Chukhrai, E.S., Itogi Nauki Tekh. Ser. Biotekhnol., 1986, vol. 5, pp. 50-86.

14. Esimbekova, E.N., Torgashina, I.G., and Kratasyuk, V.A., Biokhimiya, 2009, vol. 74, no. 6, pp. 853859.

Translated by G. Levit 